\title{
Effects of Chronic Growth Hormone Hypersecretion on Intrinsic Contractility, Energetics, Isomyosin Pattern, and Myosin Adenosine Triphosphatase Activity of Rat Left Ventricle
}

\author{
José Timsit, " Bruno Riou, „ Jérôme Bertherat," Claudine Wisnewsky," Norman S. Kato,' Andrea S. Weisberg,' \\ Jean Lubetzki," Yves Lecarpentier,"* Saul Winegrad," and Jean-Jacques Mercadier" \\ *Department of Endocrinology and "Institut National de la Santé et de la Recherche Médicale (INSERM) U 127, Hôpital Lariboisière, \\ 75010 Paris, France; ${ }^{\ddagger}$ Department of Anesthesiology, Hôpital de la Pitié-Salpétrière, 75013 Paris, France; ${ }^{8}$ INSERM U 159, \\ Centre Paul Broca, 75014 Paris, France; ${ }^{* *}$ INSERM U 275, Laboratoire d'Optique Appliquee-École Nationale Supérieure des \\ Techniques Avancées, École Polytechnique, 91120 Palaiseau, France; and 'Department of Physiology, \\ School of Medicine, University of Pennsylvania, Philadelphia, Pennsylvania 19104
}

\begin{abstract}
We studied papillary muscle mechanics and energetics, myosin phenotype, and ATPase activities in left ventricles from rats bearing a growth hormone (GH)-secreting tumor. 18 wk after tumor induction, animals exhibited a dramatic increase in body weight $(+101 \%$ vs. controls) but no change in the ventricular weight/body weight ratio. The maximum isometric force of papillary muscles normalized per cross-sectional area rose markedly $(+42 \%, P<0.05$ vs. controls), whereas the maximum unloaded shortening velocity did not change. This was observed despite a marked isomyosin shift towards V3 (32 $\pm 5 \%$ vs. $8 \pm 2 \%$ in controls, $P<0.001)$. Increased curvature of the force-velocity relationship $(+64 \%, P<0.05$ vs. controls) indicated that the muscles contracted more economically, suggesting the involvement of V3 myosin. Total calcium- and actinactivated myosin ATPase activities assayed on quickly frozen left ventricular sections were similar in tumor-bearing rats and in controls. After alkaline preincubation, these activities only decreased in tumor-bearing rats, demonstrating that V3 enzymatic sites were involved in total ATPase activity. These data demonstrate that chronic GH hypersecretion in the rat leads to a unique pattern of myocardial adaptation which allows the muscle to improve its contractile performance and economy simultaneously, thanks to myosin phenoconversion and an increase in the number of active enzymatic sites. ( $J$. Clin. Invest. 1990. 86:507-515.) Key words: acromegaly • cardiac growth • growth hormone $\cdot$ myocardial adaptation - myosin phenoconversion
\end{abstract}

\section{Introduction}

In humans, chronic hypersecretion of growth hormone (GH) is usually due to a pituitary adenoma and is responsible for

Part of this work was presented at the 62nd Scientific Sessions of the American Heart Association, New Orleans, LA, 13-16 November 1989.

Address reprint requests to Dr. Timsit, Department of Immunology and INSERM U25, Hôpital Necker, 75743 Paris, Cedex 15 France.

Received for publication 9 November 1989 and in revised form 14 February 1990

1. Abbreviations used in this paper: $\mathrm{BW}$, body weight; $\mathrm{FT}_{3}$, free triiodothyronine; GH, growth hormone; MHC, myosin heavy chain; TPF, time to peak force; TPS, time to peak shortening; VW, ventricular weight.

J. Clin. Invest.

(C) The American Society for Clinical Investigation, Inc. 0021-9738/90/08/0507/09 $\$ 2.00$

Volume 86, August 1990, 507-515 acromegaly. Chronic hypersecretion of GH, and of somatomedin C (also called IGF-I), which mediates most of the biological effects of $\mathrm{GH}$, has many morphological, functional, and metabolic consequences (1). Cardiovascular alterations consisting of cardiomegaly and hyperkinetic heart syndrome are almost always present (2-7). In many patients, heart failure occurs, and cardiovascular diseases are a major cause of death (8). Although diabetes mellitus, systemic hypertension, and coronary heart disease are especially frequent during acromegaly, alterations in cardiac function in acromegalic patients free of all these pathological conditions have suggested the existence of a cardiomyopathy specific for acromegaly (9-14).

Little is known about the pathophysiology of any such cardiomyopathy $(2,5,6,9)$. Cardiac hypertrophy has been observed in rats bearing GH-secreting transplantable tumors (15) or repeatedly treated by GH injections (16). In hemodynamic studies, conflicting observations have been reported consisting either of a hyperkinetic heart syndrome with increased cardiac output and positive derivative of the left ventricular pressure $(+\mathrm{d} P / \mathrm{d} t)$ and reduced systemic vascular resistance (15), or depressed indices of cardiac pump function with reduced $+\mathrm{d} P / \mathrm{d} t(17)$. Furthermore, these changes are indices of altered pump function of the heart, and are highly dependent upon its loading conditions, which change markedly during chronic GH hypersecretion $(7,15)$. Finally, nothing is known at present about the intrinsic contractile properties of the myocardium during this pathological condition.

Intrinsic contractility of the myocardium is defined by a whole spectrum of parameters measured on isolated muscle. One of the most important is the maximum shortening velocity of the unloaded muscle $\left(V_{\max }\right)$, because it has been shown to be related to the nature of the constituent myosin, the main protein of the sarcomere's thick filament (18). In a variety of skeletal and cardiac muscles, positive linear relationships have in fact been observed between the ability of myosin to catalyze adenosine triphosphate hydrolysis (i.e., ATPase activity), the reaction which furnishes the energy necessary for contraction, and the $V_{\max }$ of the corresponding muscles $(19,20)$. Myosin consists of a pair of heavy chains (MHC), which contain the hydrolytic enzymatic site, and two pairs of light chains, the modulatory role of which has not yet been completely established in the myocardium $(18,21)$. In the mammalian heart, two forms of MHC ( $\alpha$ and $\beta$ ) are encoded by separate genes (22). They combine to give rise to three isomyosins consisting of two homodimers, V1 $(\alpha, \alpha)$ and V3 $(\beta, \beta)$ with high and low ATPase activity, respectively, and one heterodimer V2 $(\alpha, \beta)$ with intermediate activity (23-25). All three can be easily distinguished by their electrophoretical mobility $(26,27)$. Under various physiological and pathological conditions, including 
development $(24)$ and hormonal $(26,28)$ and chronic hemodynamic alterations $(25,29-31)$, the relative proportions of these isomyosins change as a result of modifications in the expression of the corresponding genes $(32,33)$. These changes are well correlated with the alterations in the myocardial performance of the different rat and rabbit models so far studied (34-38).

Using the same left ventricular papillary muscles from rats chronically exposed to $\mathrm{GH}$ hypersecretion, we studied both the intrinsic contractility and the relative proportions of the three isomyosins. We also measured the calcium- and actin-activated myosin ATPase activities in cryostatic sections of quickly frozen left ventricles from a similar group of rats. This was done because myosin ATPase activities measured in this way are more likely to reflect the in vivo function of the active enzymatic sites modulated by their regulatory systems than methods using isolated myofibrills or purified proteins (21, 39). Our results show that chronic GH hypersecretion was associated with an increase in papillary muscle active force normalized per cross-sectional area and no change in both the unloaded shortening velocity of the isolated muscles and the calcium- and actin-activated myosin ATPase activities. This contrasted strongly with a marked shift of the isomyosin pattern towards the low ATPase activity V3 isoform.

\section{Methods}

Rat model of chronic GH hypersecretion. Care of the animals and conduct of all experiments were in accordance with the recommendations of the Helsinki Guidelines. GC cells were cultured in Ham's F10 medium supplemented with $15 \%$ horse serum and $2.5 \%$ fetal calf serum (Gibco-BRL, Cergy-Pontoise, France). A suspension of 10-15 $\times 10^{6}$ cells in Hanks' medium $(0.3 \mathrm{ml} \mathrm{vol})$ was injected subcutaneously into the flank of 10-12-wk-old female Wistar Furth rats (Iffa Credo, L'Arbresle, France) under sodium metohexitone anesthesia $(40 \mathrm{mg} / \mathrm{kg}$ i.p.). Animals were weighed weekly. Tumor-bearing rats were divided into three groups. Group Al $(n=5)$ was studied $10 \mathrm{wk}$ after injection, and group A2 $(n=4) 18 \mathrm{wk}$ after injection. In group A3 $(n=4)$, the tumor was resected under anesthesia $10 \mathrm{wk}$ after injection and the animals were studied 8 wk later (i.e., 18 wk after injection). Corresponding groups of control littermates ( $n=6,5$, and 5, respectively), were also studied.

Rats were anesthetized i.p. with $30 \mathrm{mg} / \mathrm{kg}$ sodium pentobarbital, weighed, and killed. Blood was collected through the inferior vena cava. The heart was quickly removed and the two left ventricular papillary muscles were carefully excised for the mechanical study. The heart was washed in ice-cold saline and dissected free of the atria. Ventricles were weighed and frozen immediately in liquid nitrogen, and the two papillary muscles were also frozen at the end of the mechanical study in order to determine their isomyosin patterns. Blood was centrifuged and plasma was frozen for hormonal assays.

A fourth group of tumor-bearing rats $(\mathrm{A} 4, n=5)$ and their controls $(n=4)$ were used to study calcium- and actin-activated myosin ATPase activities in sections of the left ventricle. $18 \mathrm{wk}$ after injection of the cell suspension, they were killed by decapitation. In $<1$ min the heart was excised and washed, the right ventricle was removed, and the left ventricle was frozen in isopentane previously cooled in liquid nitrogen (39).

Hormonal assays. Plasma GH and prolactin concentrations were determined by radioimmunoassay (RIA) using reagents provided by the National Institute of Arthritis, Digestive Diseases, and Kidney as described (40). The free fraction of triiodothyronine $\left(\mathrm{FT}_{3}\right)$ was measured using a commercially available kit (FT ${ }_{3}$-RIAsol, Henning, $\mathrm{GmbH}$, Berlin, FRG). All determinations were done in duplicate.
Papillary muscle mechanics. < 1 min after rats were killed, left ventricular papillary muscles were suspended vertically in a $60-\mathrm{ml}$ Krebs-Henseleit bicarbonate buffer solution containing $\mathrm{CaCl}_{2} 6 \mathrm{H}_{2} \mathrm{O}$ $2.5 \mathrm{mM}$, pH 7.40 (41). Temperature was maintained at $+29^{\circ} \mathrm{C}$. Muscles were field-stimulated at $0.12 \mathrm{~Hz}$ by means of two platinum electrodes with rectangular wave pulses of 5-ms duration and amplitude just above threshold. The electromagnetic level system has already been described (41). The system had a linear range up to $2.5 \mathrm{~mm}$ of muscle shortening (resolution $<0.1 \mathrm{~mm}$ ). The amplitude of the force ranged from 0 to $100 \mathrm{mN}$ (resolution $<0.1 \mathrm{mN}$ ). The equivalent moving mass of the whole system was $155 \mathrm{mg}$, and the compliance was $0.2 \mu \mathrm{m} / \mathrm{mN}$. After a 1-h stabilization period at $L_{\max }$ (i.e., the initial muscle length corresponding to the apex of the length-active isometric tension relationship), papillary muscles had recovered their optimal mechanical performance. All analyses were made from digital records obtained with a computer (model 1000, Hewlett-Packard Co., Palo Alto, CA).

Mechanical parameters at $L_{\max }$ were calculated from three types of twitches. The first twitch was isotonic and was loaded only with the preload corresponding to $L_{\max }$ (twitch 1 ). In the second twitch this preload was abruptly removed $3 \mathrm{~ms}$ after the electrical stimulus according to the zero-load clamp technique $(37,42)$ (twitch 2 ). The third twitch was fully isometric at $L_{\max }$ (twitch 3 ). The mechanical parameters characterizing the contraction phase were defined as follows: maximum extent of shortening $(\Delta L)$ from twitch $1 ; V_{\max }$ determined from twitch 2; maximum isometric active force normalized per crosssectional area $(A F / s)$ and its rate of rise $(+\mathrm{d} F / \mathrm{d} t$ per $s)$ determined from twitch 3; time to peak shortening of twitch 1 (TPS) and time to peak force of twitch $3(T P F)$. Shortening and lengthening velocities were expressed in $L_{\max } \cdot \mathrm{s}^{-1}, T P S$ and $T P F$ in milliseconds, and force in $\mathrm{mN} \cdot \mathrm{mm}^{-2}$.

Papillary muscle energetics. Energetic parameters were derived from the total force-velocity relationship. This relationship was established from the peak shortening velocity of seven to nine twitches of each papillary muscle, whose load was regularly increased from zero to full isometric load. Data were fitted by means of multiple linear regression analysis and the least square method, according to Hill's equation (43): $(T F / s+a)(V+b)=\left(T F_{\max } / s+a\right) b$, where $T F / s$ is the total isotonic force, $T F_{\max } / s$ the maximal value of $T F / s$ when the twitch is fully isometric, $V$ the peak shortening velocity of the isotonic twitch, and $-a$ and $-b$ the asymptotes of the hyperbola. This allowed calculation of the $G$ curvature of the force-velocity relationship ( $G$ $=V_{\max } / b=T F_{\max } / s$ per $a$ ), and $M$ which is the positive root of the quadratic equation: $G M^{2}+2 M-1=0(37,44)$. The peak power output

$\dot{E}_{\max }=M^{2} \times V_{\max } \times T F_{\max } / s$,

where $M^{2}$ is the normalized peak power output. The product $a \times b$ has been found to represent the rate of maintenance heat production (43, 45). From a thermoenergetic point of view, $G$, the curvature of the force-velocity relationship has been proposed to reflect the efficiency of muscle contraction $(37,44,46)$. The higher the $G$ value, the higher the myothermal economy of contraction.

Isomyosin pattern. The isomyosin pattern was determined for each papillary muscle, and for the epicardial half of the left ventricular free walls of the rats in group A4, as previously described (30). Muscles were minced with scissors and extracted for $20 \mathrm{~min}$ with $4 \mathrm{vol}$ of slightly modified ice-cold Guba-Straub's solution. The proportion of each myosin form was determined by scanning after separation by electrophoresis under nondenaturing conditions on cylindrical polyacrylamide gels in the presence of pyrophosphate. Results were expressed both as the percentage of each V1, V2, and V3 isoform and as the percentage of $\beta$-MHC, taking into account that V2 is an $\alpha \beta$-heterodimer.

Myosin ATPase activity. Left ventricles from group A4 rats were attached to the stub of a Hacker cryostat and 4- $\mu \mathrm{m}$ sections of the subepicardium were cut. The calcium- and actin-activated myosin 


\begin{tabular}{|c|c|c|c|c|c|}
\hline & \multicolumn{5}{|c|}{ Group } \\
\hline & $C(n=16)$ & Al $(n=5)$ & & $\mathrm{A} 2(n=4)$ & $\mathrm{A} 3(n=4)$ \\
\hline BW $(g)$ & $217 \pm 6$ & $417 \pm 2^{*}$ & & $436 \pm 14^{*}$ & $324 \pm 14^{* \neq 8}$ \\
\hline $\mathrm{VW}(m g)$ & $637 \pm 35$ & $1,181 \pm 56^{*}$ & & $1,222 \pm 32^{*}$ & $942 \pm 46^{* 16}$ \\
\hline Left VW $(m g)$ & $497 \pm 27$ & $937 \pm 42^{*}$ & & $908 \pm 36^{*}$ & $749 \pm 38^{* \pm \S}$ \\
\hline Left VW/BW & $2.29 \pm 0.11$ & $2.25 \pm 0.09$ & . & $2.08 \pm 0.11$ & $2.31 \pm 0.04$ \\
\hline TW $(g)$ & - & $10.7 \pm 5.6$ & & $12.9 \pm 3.5$ & - \\
\hline $\mathrm{GH}(n g / m l)$ & $20 \pm 22$ & $1,116 \pm 448^{*}$ & & $1,915 \pm 380^{*}$ & $28 \pm 18^{\ddagger \S}$ \\
\hline $\mathrm{FT}_{3}(p M /$ liter $)$ & $4.14 \pm 1.58$ & $3.44 \pm 2.66$ & & $0.55 \pm 0.70^{*}$ & $2.95 \pm 2.86$ \\
\hline
\end{tabular}

Female Wistar-Furth rats were studied $10 \mathrm{wk}$ (A1), and $18 \mathrm{wk}$ (A2) after subcutaneous injection of GH-secreting GC cells. In group A3, tumors were allowed to grow for 10 wk and were then resected. Rats were studied after an 8-wk recovery period. GH and $\mathrm{FT}_{3}$ were measured by RIA. Results are means \pm SD. C, control. ${ }^{*} P<0.05$ vs. $C ;{ }^{\ddagger} P<0.05$ vs. A2; ${ }^{8} P<0.05$ vs. A1.

ATPase activities were measured by quantitative microphotometry as previously described $(39,47)$. The principle of the method consists of trapping the inorganic phosphate produced by ATPase activity with calcium ions, and the quantitative replacement of the calcium phosphate by the more opaque cobalt sulfide. The optical density (OD) in each of 10 alternate serial sections of each left ventricle was measured on a digitized image, using a TV camera attached to a photomicroscope (Carl Zeiss, Inc., Oberkuchen, FRG). The calcium- or actin-activated myosin ATPase activity of each left ventricle is proportional to the mean $O D$ values measured in each of the 10 sections. The standard error was $<2 \%$. In each experiment, the left ventricles of a tumorbearing and of a control animal were studied. The calcium- and actinactivated myosin ATPase activities were measured in the presence of ouabain and azide to inhibit the $\mathrm{Na}^{+} / \mathrm{K}^{+}$- and mitochondrial ATPases, respectively. Myosin ATPase activities were assayed both after preincubation at neutral $\mathrm{pH}$, which allows the determination of the total ATPase activity, and after inhibition of the V3 isomyosin by preincubation at alkaline $\mathrm{pH}(10.3)$ in the absence of ATP, which permits the measurement of the ATPase activity due to V1 only $(23,39,47)$. Relative ATPase activities are indicated by absorbance units. The myosin phenotype was determined in the subepicardium of the left ventricles, exactly where the sections had been cut.

Statistical analysis. Data were expressed as means \pm SD. Control groups were compared by one-way analysis of variance. As no differences in mechanical, biochemical or hormonal parameters were observed, the control groups (16 rats, 31 papillary muscles) were pooled. Groups C, A1, A2, and A3 were compared by one-way analysis of variance and Student's $t$ test with the Bonferroni correction. For the myosin ATPase activity study, paired and unpaired Student's $t$ tests were used as appropriate. All comparisons were two-tailed and a $P$ values $<0.05$ were considered significant.

\section{Results}

Effects of tumor growth on rat body weight $(B W)$, ventricular weight $(V W)$, and plasma hormone levels. Tumor growth occurred in $90 \%$ of injected rats. BW increased as a function of time in the rats of groups $A 1$ and $A 2$, whereas it plateaued at about $220 \mathrm{~g}$ in the controls (Table I). In group A3, rat BW increased similarly during the first $10 \mathrm{wk}$ postinjection up to $424 \pm 14 \mathrm{~g}$, but dropped sharply to $333 \pm 19 \mathrm{~g}$ soon after tumorectomy. However, at $18 \mathrm{wk}$, the BW of these animals remained higher than that of their age-matched controls $(324 \pm 14$ vs. $216 \pm 6 \mathrm{~g}, P<0.001$ ). VW was also greater in the three groups of tumor-bearing animals than in controls but the VW/BW and left VW/BW ratios were not different from control ratios
(Table I), indicating that no cardiac hypertrophy (i.e., no increase in the heart weight to BW ratio) occurred in tumorbearing rats. Left ventricular papillary muscles were longer in the rats of groups $\mathrm{A} 1$ and $\mathrm{A} 2$ than in the controls, but the cross-sectional area was significantly larger in group A1 only (Table II). As expected, GH plasma levels were dramatically higher in tumor-bearing rats than in controls, and about twice as high in group A2 as in group A1. By contrast, in group A3, GH plasma levels were normal $8 \mathrm{wk}$ after tumorectomy (Table I). The plasma $\mathrm{FT}_{3}$ concentration was not different in groups A1 and A3 from that of the controls, but was lower in group A2. Prolactin plasma concentration did not increase in any of the three injected groups of rats compared to their controls (data not shown).

Papillary muscle mechanics. Fig. 1 shows characteristic traces of force and shortening as a function of time in a papillary muscle contracting against various loads from a control and a tumor-bearing rat in group A2. The main mechanical parameters derived from these traces are indicated in Table III. The maximum shortening velocity of the unloaded muscle $\left(V_{\max }\right)$, did not differ from control values in any experimental group of rats. By contrast, the active force normalized per cross-sectional area $(A F / s)$, which was already increased, but not significantly, in group Al compared to the controls, rose markedly in group A2 (Fig. 2 and Table III, $+42 \%, P<0.05$ vs. controls). If, as is generally accepted, each cycling cross-bridge develops the same elementary force (45), the increase in $A F / s$ in tumor-bearing rats requires that the number of functional

Table II. Physical Characteristics of Left Ventricular Papillary Muscles from Control and Experimental Rats

\begin{tabular}{lcccc}
\hline & \multicolumn{4}{c}{ Group } \\
\cline { 2 - 5 } & $\mathrm{C}(n=31)$ & $\mathrm{A} 1(n=10)$ & $\mathrm{A} 2(n=8)$ & $\mathrm{A} 3(n=8)$ \\
\hline$L_{\max }(\mathrm{mm})$ & $4.7 \pm 1.1$ & $6.0 \pm 1.6^{*}$ & $5.9 \pm 1.2^{*}$ & $5.4 \pm 1.2$ \\
$\mathrm{~s}\left(\mathrm{~mm}^{2}\right)$ & $0.77 \pm 0.15$ & $0.98 \pm 0.22^{*}$ & $0.90 \pm 0.15$ & $0.86 \pm 0.16$
\end{tabular}

Animal groups are the same as in Table I. C, control; $L_{\max }$, length of papillary muscles preloaded for maximal active isometric force; $s$, cross-sectional area of papillary muscles. Values are means $\pm \mathrm{SD} .{ }^{*} P$ $<0.05$ vs. C. 

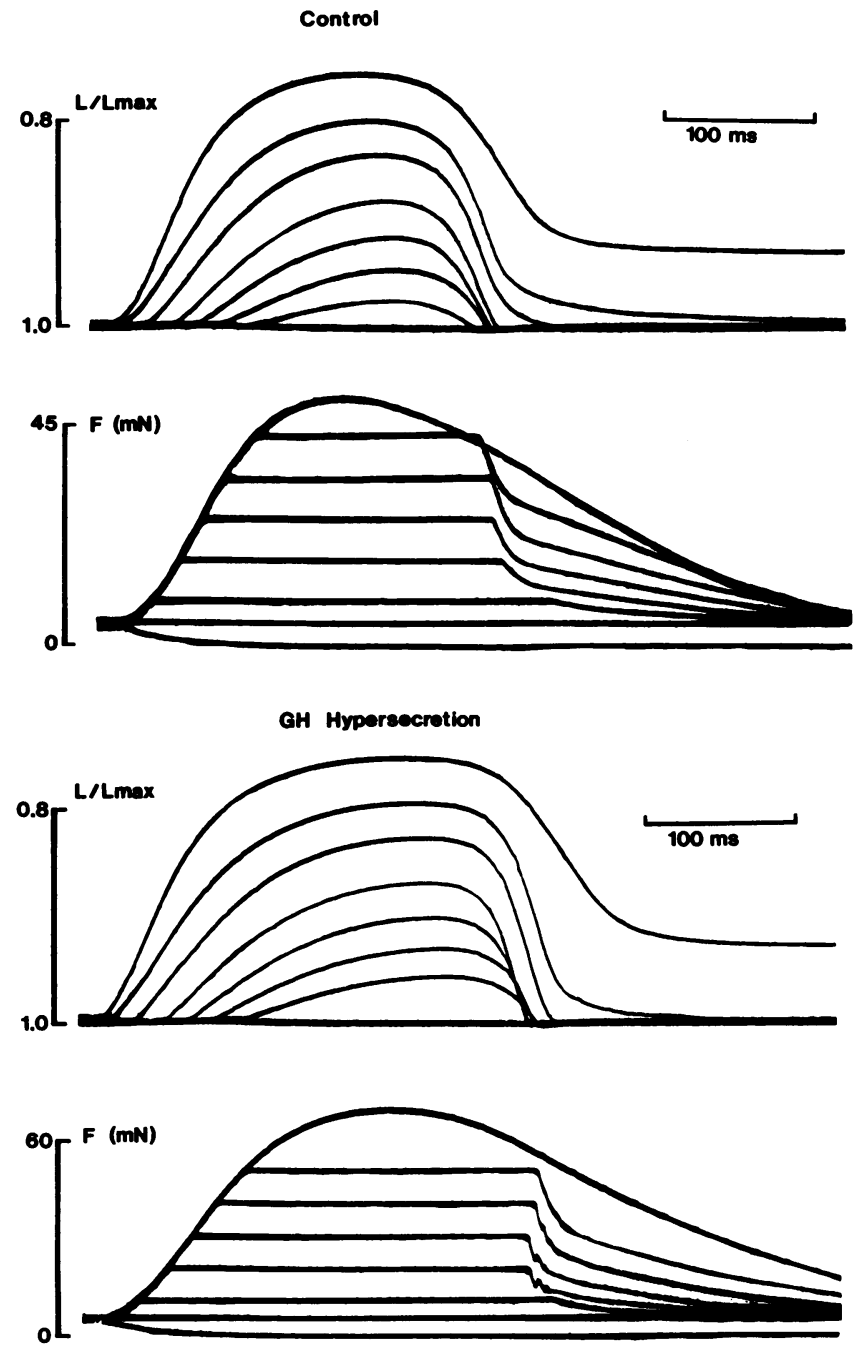

Figure 1. Typical traces of left ventricular papillary muscle contraction from a control rat (top) and a GH-secreting tumor-bearing rat from group A2 (bottom). Upper graph: muscle shortening $\left(L / L_{\max }\right)$ plotted vs. time. Lower graph: force $(F)$ plotted vs. time. Time to peak shortening (TPS), time to peak force (TPF), and total isometric force of papillary muscle are higher in group A2 than in controls.

cross-bridges per unit of cross-sectional area increased in tumor-bearing rats compared to controls. The rate of rise of $A F / s$ (i.e., $+\mathrm{d} F / \mathrm{d} t$ per $s$ ) tended to increase in groups $\mathrm{Al}$ and
A2 vs. the controls, but this did not reach statistical significance. The temporal mechanical parameters TPS and TPF did not change in group A1 but rose in group A2 compared to the controls (Table III, $+18 \%, P<0.05$ and $+21 \%, P<0.05$, respectively). In group $A 3$, removal of the tumor after 10 weeks prevented the mechanical alterations observed in group A2.

Papillary muscle energetics. The parameters which account for the energetics of papillary muscles contraction are given in Table IV. $G$, the curvature of the force-velocity relationship progressively increased in groups $\mathrm{A} 1$ and $\mathrm{A} 2$ vs. the controls. This increase did not reach statistical significance in group A1, but was dramatic in group A2 $(+64 \%, P<0.05$ vs. controls). This increase suggested that papillary muscles of tumor-bearing rats contracted more economically than control muscles. As a consequence of the increase in $G, M^{2}$, the normalized peak power output, decreased in tumor-bearing rats. In group A2, this decrease was $16 \%$ of the control value $(P$ $<0.05$ ). It was interesting to observe that the peak power output $E_{\max }$ did not change in any group of tumor-bearing rats. This can be explained by the decrease in $M^{2}$ which roughly compensated the increase in $T F_{\max } / s$, whereas $V_{\max }$ did not change (see Eq. 1 in Methods). In the A2 group, only the b coefficient decreased significantly. This decrease, combined with an unsignificant decrease in the a coefficient, resulted in a decrease of about $50 \%$ in the calculated isometric maintenance heat rate $(a \times b)(P<0.05$ vs. controls). In group $\mathrm{A} 3$, the alterations observed in muscle energetics in group A2 were prevented by tumor resection.

Isomyosin pattern of papillary muscles. Typical gels of a papillary muscle from each of the four groups of rats are shown in Fig. 3. The relative proportions of V1, V2, and V3 isomyosins are shown in Fig. 4. In control rats, V1 was the predominant isoform ( $64 \pm 3 \%$ of total myosin) and V3 was only $8 \pm 2 \%$. Together with V2, this corresponded to $22 \pm 2 \% \beta$-MHCs. A decrease in V1 $(54 \pm 2 \%, P<0.01$ vs. controls) was observed in muscles from group A1 rats and a parallel increase in V3 ( $19 \pm 2 \%, P<0.01$ vs. controls). A dramatic shift was observed in $\mathrm{A} 2$ rats in which $\mathrm{V} 1$ dropped to $34 \pm 5 \%$ and $\mathrm{V} 3$ rose to $32 \pm 5 \%$ ( $P<0.001$ vs. controls for all comparisons). Together with V2, this corresponded to $49 \pm 5 \% \beta$-MHCs. Consequently A 2 rats had $27 \%$ more $\beta$-MHCs than controls. In A3 rats, the proportions of each isoform were very similar to those of group Al $(50 \pm 4 \%$ and $18 \pm 3 \%$ for V1 and V3, respectively, NS vs. group A1 for all comparisons). Thus, tumorectomy at $10 \mathrm{wk}$, which was associated with a large, fast drop in BW and with

Table III. Mechanical Parameters of Left Ventricular Papillary Muscles from Control and Experimental Rats

\begin{tabular}{lcccc}
\hline & \multicolumn{3}{c}{ Group } \\
\cline { 2 - 5 } & $\mathrm{C}(n=31)$ & $\mathrm{A} 1(n=10)$ & $\mathrm{A} 2(n=8)$ & A3 $(n=8)$ \\
\hline$V_{\max }\left(L_{\max } \cdot \mathrm{s}^{-1}\right)$ & $3.24 \pm 0.38$ & $3.05 \pm 0.35$ & $3.09 \pm 0.21$ & $3.32 \pm 0.15$ \\
$\mathrm{AF} / \mathrm{s}\left(m N \cdot m m^{-2}\right)$ & $48.5 \pm 15.8$ & $57.7 \pm 20.4$ & $69.1 \pm 22.9 *$ & $56.4 \pm 17.9$ \\
$+d \mathrm{~F} / \mathrm{d} t / s\left(m N^{-1} \cdot \mathrm{mm}^{-2}\right)$ & $561 \pm 230$ & $678 \pm 246$ & $711 \pm 253$ & $587 \pm 205$ \\
$\mathrm{TPS}(m s)$ & $189 \pm 15$ & $185 \pm 7$ & $223 \pm 10^{* \neq \S}$ & $199 \pm 13$ \\
TPF $(m s)$ & $174 \pm 16$ & $170 \pm 13$ & $211 \pm 20^{* \neq \S}$ & $187 \pm 18$
\end{tabular}

Animal groups are the same as in Table I. C, control; $V_{\max }$, maximum unloaded shortening velocity; AF/s, active force normalized per crosssectional area; $+\mathrm{d} F / \mathrm{d} t / s$, rate of rise of $\mathrm{AF} / \mathrm{s}$; TPS, time to peak shortening; TPF, time to peak force. Values are means $\pm \mathrm{SD} ;{ }^{*} P<0.05 \mathrm{vs}$. $\mathrm{C}$; ${ }^{\ddagger} P<0.05$ vs. $\mathrm{A} 1 ;{ }^{\S} P<0.05$ vs. A3. 


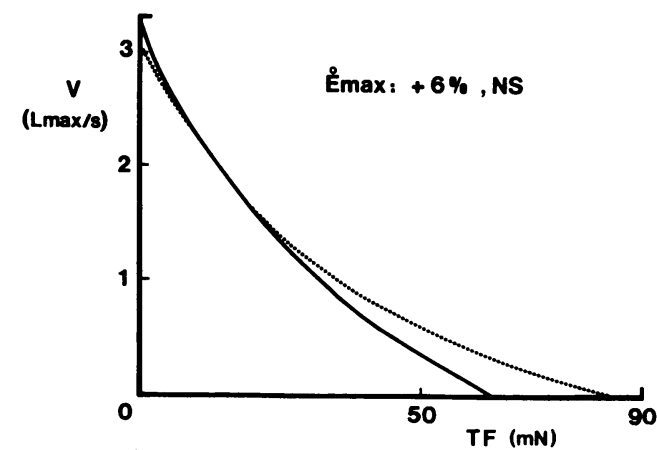

normalization of GH plasma levels, was not associated with normalization of the isomyosin pattern, but rather prevented the shift observed in the animals bearing the tumor for $18 \mathrm{wk}$.

In group A4, a marked isomyosin shift was observed as in the other tumor-bearing rats (Table V). Indeed, in the subepicardium, V3 constituted $16 \pm 4 \%$ of total myosin, whereas no V3 was detected in that of control rats. These percentages were lower than those measured in the papillary muscles of tumorbearing rats and their controls. This could be explained by the regional variations in ventricular isomyosin distribution previously reported $(31,48)$. Together with $35 \pm 4 \% \mathrm{~V} 2$, the $16 \pm 4 \% \mathrm{~V} 3$ in the subepicardium of group A4 rats yielded $33 \%$ $\beta$-MHCs compared with $0 \%$ in their controls (Table V). This difference in the percentage of $\beta$-MHC was approximately the same as that observed between the papillary muscles of rats in group A2 and their controls (27\%).

Calcium-and actin-activated myosin ATPase activities. Most interestingly, despite the profound isomyosin shift observed in the subepicardium of the left ventricles in group A4 rats compared with controls, both calcium- and actin-activated myosin ATPase activities at neutral preincubation were almost exactly identical in the two groups of rats (Table V). This demonstrated that the net effect of the functional enzymatic sites was the same in normal and tumor-bearing rats. By contrast, alkaline preincubation significantly decreased the levels of both calcium- and actin-activated myosin ATPase activities only in group A4 rats $(-6 \%$ and $-19 \%$ of the corresponding values at neutral $\mathrm{pH}$, respectively, $P<0.05$ for both comparisons). This demonstrated that the ATPase activity levels observed at neutral preincubation in group A4 were due in part to the presence of functional enzymatic sites sensitive to alkaline preincubation, i.e., to sites consisting of $\beta$-MHCs, which was not the case in control rats. Considering that the ATPase activity of V3 is about one-third that of V1 $(23,24,49)$ and that total ATPase activities at neutral $\mathrm{pH}$ were identical in tumor-bearing rats and in their controls, it is possible to write the following simplified equation:

$k \cdot N_{\mathrm{A}}+0.3 k \cdot N_{\mathrm{B}}=k \cdot N_{\mathrm{C}}$,

where $k$ is the ATPase activity of a V1 myosin head, $N_{\mathrm{A}}$ and $N_{\mathrm{C}}$ are the number of active $\mathrm{V} 1$ enzymatic sites in tumor-bearing and control rats, respectively, and $N_{\mathrm{B}}$ the number of active V3 enzymatic sites in tumor-bearing rats, with $N_{\mathrm{B}}>0$ as demonstrated by alkaline preincubation. Eq. 2 implies the recruitment, in A4 rats, of an increased total number of functional enzymatic sites, since the increase in the number of functional V3 sites must be higher than the decrease in the amount of functional V1 sites to preserve normal ATPase activities. Thus, during chronic GH hypersecretion in rats, normal ventricular myosin ATPase activities were observed despite the marked myosin phenoconversion, owing to the recruitement of an increased number of functionally active enzymatic sites.

\section{Discussion}

Our study is the first to assess the intrinsic contractile properties of the myocardium in rats subjected to chronic GH hyper-

Table IV. Comparison of Energetic Parameters in the Left Ventricular Papillary Muscles of Control and Experimental Rats

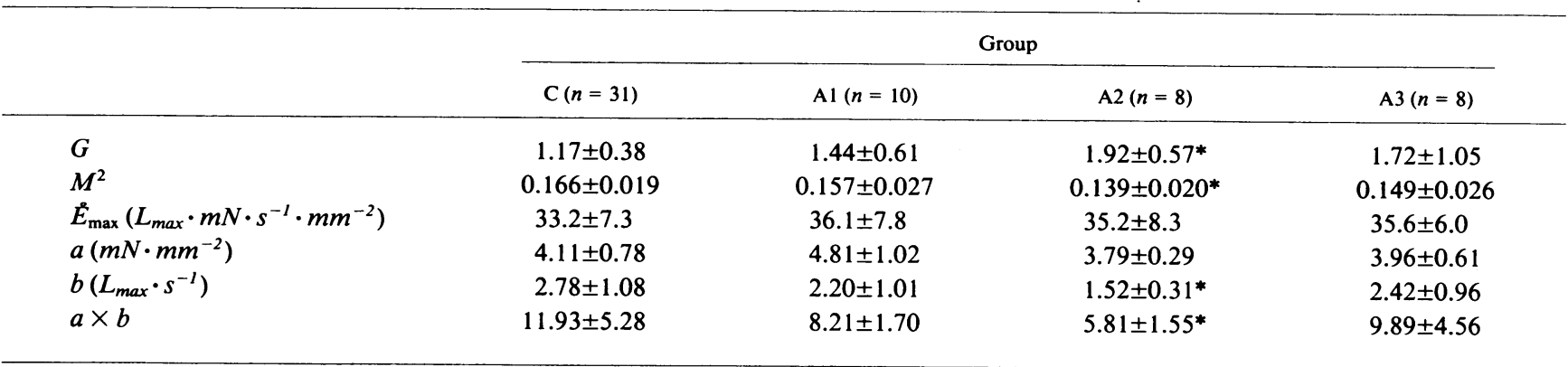

Animal groups are the same as in Table I. C, control; $G$, curvature of the force-velocity relationship; $M^{2}$, normalized peak power output; $E_{\text {max }}$, peak power output; $a$ and $b$, constants of the hyperbola. Values are means $\pm \mathrm{SD} ;{ }^{*} P<0.05$ vs. C. 


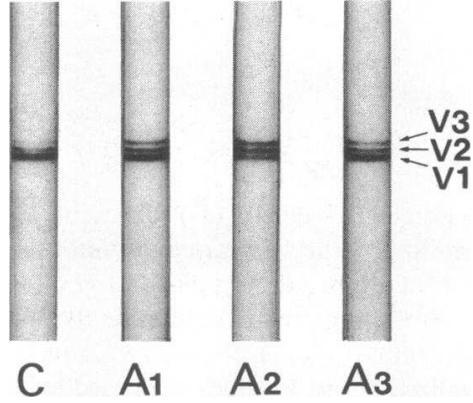

Figure 3. Typical electrophoretic patterns under nondenaturing conditions of myosins from the left ventricular papillary muscles of a control rat (C), and from GH-secreting tumor-bearing rats in groups $\mathrm{A} 1, \mathrm{~A} 2$, and $\mathrm{A} 3$.

secretion, and to determine the myosin phenotype in the same left ventricular papillary muscles, the necessary condition for rigorous examination of the relationships between the mechanical and biochemical parameters of muscle contraction. Furthermore, in another group of rats, we measured the calcium- and actin-activated myosin ATPase activities and the corresponding isomyosin pattern in the left ventricular free wall. The results clearly show that in tumor-bearing animals, normal or supranormal myocardial contractility was associated with normal myosin ATPase activities, despite a marked phenoconversion of myosin toward V3. Thus, our separate mechanical and enzymatic studies consistently demonstrated that chronic GH hypersecretion in rats induced a unique pattern of myocardial contraction: a normal shortening speed and an increased force generation are associated with changes in myosin phenotype that allow the muscle to function more economically.

The existence of alterations in cardiac function in human subjects submitted to chronic GH hypersecretion has been known for a long time (8). In most patients with acromegaly, cardiac hypertrophy is present and is associated with an hyperkinetic heart syndrome $(3,7)$. In both $\mathrm{GH}$-deficient patients $(50,51)$ and normal volunteers (52), treatment with recombinant $\mathrm{GH}$ has been suggested to increase myocardial performance and cardiac output. Conversely, in acromegalic patients, treatment with octreotide, the somatostatin analogue, reduced left ventricular mass and echographic indices of myocardial contractility (53). Few studies have assessed cardiac

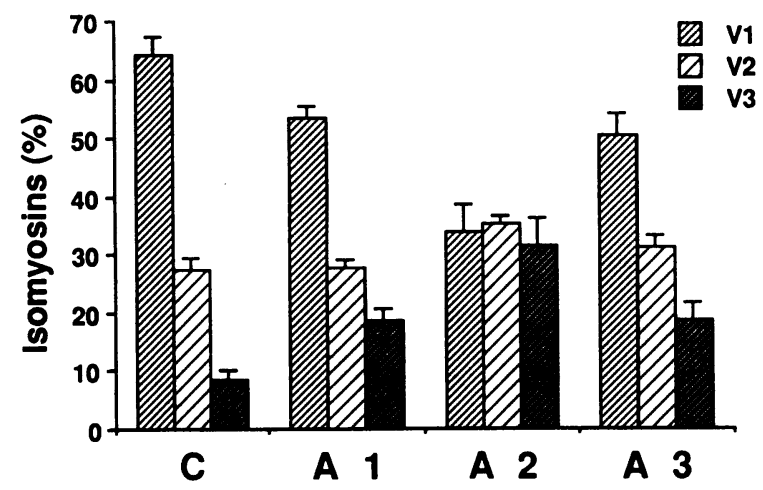

Figure 4. Mean percentage of each myosin form in left ventricular papillary muscles from control rats $(\mathrm{C}, n=13)$, and from GH-secreting tumor-bearing rats in groups $\mathrm{A} 1(n=9), \mathrm{A} 2(n=8)$, and $\mathrm{A} 3(n$ $=7$ ). Bars represent SD. $P<0.01$ for comparisons of $V 1 \%$ and $V 3 \%$ in groups $\mathrm{A} 1, \mathrm{~A} 2$, and $\mathrm{A} 3$ vs. $\mathrm{C}$, in group $\mathrm{A} 2$ vs. $\mathrm{A} 1$, and in group $A 3$ vs. A2; NS for the same comparisons of $A 3$ vs. A1.
Table V. Myosin ATPase Activities and Isoforms in Left Ventricles from Control and Experimental Rats

\begin{tabular}{lcc}
\hline & \multicolumn{2}{c}{ Group } \\
\cline { 2 - 3 } & $\mathrm{C}(n=4)$ & $\mathrm{A4}(n=5)$ \\
\hline $\mathrm{BW}(g)$ & $220 \pm 17$ & $442 \pm 48^{*}$ \\
Left VW $(m g)$ & $599 \pm 5$ & $1056 \pm 136^{*}$ \\
Left VW/BW & $2.73 \pm 0.21$ & $2.40 \pm 0.24$ \\
$\mathrm{Ca}^{2+}$-activated myosin ATPase & & \\
$\quad$ Neutral preincubation & $0.439 \pm 0.032$ & $0.433 \pm 0.042$ \\
$\quad$ Alkaline preincubation & $0.441 \pm 0.033$ & $0.405 \pm 0.046^{\ddagger}$ \\
Actin-activated myosin ATPase & & \\
$\quad$ Neutral preincubation & $0.148 \pm 0.012$ & $0.154 \pm 0.032$ \\
Alkaline preincubation & $0.133 \pm 0.027$ & $0.125 \pm 0.027^{\ddagger}$ \\
V1 (\%) & $100 \pm 0$ & $49 \pm 7^{*}$ \\
V2 (\%) & $0 \pm 0$ & $35 \pm 4^{*}$ \\
V3 (\%) & $0 \pm 0$ & $16 \pm 4^{*}$ \\
$\beta-M H C(\%)$ & $0 \pm 0$ & $33 \pm 5.7^{*}$
\end{tabular}

Group A4, tumor-bearing rats studied 18 wks after GC cell injection; C, control rats. Myosin ATPase activities of left ventricular sections were measured after neutral or alkaline preincubation (see Methods). Percentages of myosin isoforms (V1, V2, and V3) were determined by pyrophosphate gel analysis. Values are means \pm SD. ${ }^{*} P<0.001$ vs. $C ;{ }^{\ddagger} P<0.05$ vs. neutral preincubation.

function in experimental models of chronic GH hypersecretion. In the most documented hemodynamic study in the rat, the alterations reported were clearly consistent with the hyperkinetic heart syndrome observed in man; thus, increases in cardiac index, $+\mathrm{d} P / \mathrm{d} t$ max, and aortic blood flow were combined with normal arterial pressure and a decrease in systemic vascular resistance (15). Since hemodynamic studies give little direct information about the intrinsic contractile properties of the myocardium, our goal has been to assess the various mechanical and biochemical parameters which characterize myocardial contractile function in this peculiar model of cardiac growth.

We used the model of the tumor derived from GC cells which secretes large amounts of biologically active $\mathrm{GH}$, as demonstrated by plasma $\mathrm{GH}$ levels, which were 50-100 times higher in tumor-bearing rats than in controls, and by the marked overgrowth of these animals. A number of procedures resulting in a chronic increase in plasma $\mathrm{GH}$ have been described in small mammals, including daily GH injection (16), implantation of tumors secreting $\mathrm{GH}$ and prolactin $(15,54)$, and transfection of mice oocytes with the $\mathrm{GH}$ or GH-releasing hormone gene $(55,56)$. Our model offers several advantages: it is easily produced and the removal of the subcutaneous tumor allows study of the response to termination of $\mathrm{GH}$ hypersecretion at different stages. In addition, the absence of excessive secretion of prolactin by the GC cell-derived tumor eliminates the many known effects of this hormone, particularly on the water and sodium balance (57).

We did not observe any cardiac hypertrophy in the rats exposed to chronic excess of GH. Although we observed considerable enlargement of both ventricles in the three groups compared with the controls, neither the $\mathrm{VW} / \mathrm{BW}$ ratio nor the ratio of each ventricle's weight to body weight altered. In a previous study of rats bearing the MtT-W-15 GH-secreting 
tumor (15), a $33 \%$ increase in the $\mathrm{VW} / \mathrm{BW}$ ratio was observed in rats with "large" tumors compared to the controls, essentially to the benefit of the right ventricle. The reason for this discrepancy is not clear, since in both studies the duration and level of GH hypersecretion were essentially the same. By contrast our results are in full agreement with the study by Gilbert et al. (54), who used a model close to ours. They found a normal VW/BW ratio in tumor-bearing rats, although the plasma GH levels they measured were even higher than those observed here. In detailed pathological studies carried out by the same group, the hearts only exhibited an increase in myocyte length (58). These and our results therefore seem to indicate that chronic GH hypersecretion in rat is associated with a growth of the heart proportional to the growth of the body. We also observed that tumor-bearing rats had both normal $V_{\max }$ and increased $A F / s$. This is in sharp contrast with what occurs in all models of chronic hemodynamic overload which result in ventricular hypertrophy, associated with a decrease in $V_{\max }$ and $A F / s(34,35,37,38,46)$.

The most striking finding in our study was the observation that normal or supra-normal contractile performance in tumor-bearing rats was associated with a marked isomyosin shift towards V3. To our knowledge, this is the first demonstration in the adult rat of a dissociation between myocardial myosin phenotype and contractility. Chronic hemodynamic overload, aging, and hypothyroidism are associated with a decreased $V_{\max }$ and an increased proportion of $\mathrm{V} 3$, whereas the opposite is seen in young animals and in hyperthyroid or old animals treated by thyroid hormone. Strong linear correlations were found between the proportions of each one of the two main myosin isoforms and $V_{\max }$, in rats exhibiting these various physiological and pathological conditions (18, 34, 36-38). According to these correlations, the $34 \% \mathrm{~V} 1$ and $32 \% \mathrm{~V} 3$ that we measured in the papillary muscles of the rats in group A2 compared with the $64 \%$ V1 and $8 \%$ V3 in the controls should have been associated in tumor-bearing rats, with a decrease of $V_{\max }$ of at least $30 \%$, which we did not in fact observe. It is also worthwhile to note that during chronic hemodynamic overload in which myosin phenoconversion towards V3 is observed, $A F / s$ is decreased in contrast to the marked increase observed here $(34,37,38)$. Myosin phenoconversion we observed in group A2 rats might be explained in part by the lower $\mathrm{FT}_{3}$ plasma concentration noted in these rats. However, a significant shift towards V3 also occurred in the animals of group A1, which had normal $\mathrm{FT}_{3}$. These data are fully consistent with the results reported for rats injected with $\mathrm{GH}$ for $5 \mathrm{wk}$, whose $\mathrm{V} 1$ in the left ventricle decreased by $25 \%$ while their plasma $T_{3}$ levels were normal (16). Moreover, hypothyroidism is associated with a great reduction in $V_{\max }(34,35)$ and $A F / s$ $(34,49)$, which we did not observe in our model.

One of the important points in this study is the consistency between the results of the mechanical, energetical and enzymatic studies. Moreover, the relations that can be established between several of the measurements performed bring some insight into the alterations which have occurred in the mechanisms of myocardial contraction of tumor-bearing rats. It is generally agreed in the isometric twitch, that the maximum active force is a function of the number of active cross-bridges $(44,45,59,60)$. In our study, the $42 \%$ increase in $A F / s$ in group A2 (Table III), indicates an increase in the number of active cross-bridges. The results of the enzymatic study sup- port this conclusion. Since V3 has an ATPase activity $\sim 0.3$ of V1 $(23,24,49)$, the constant total ATPase activity in tumorbearing rats despite the contribution by V3 (assessed by the appearance of sizable alkaline labile ATPase activity), shows that a larger number of cross-bridges were active, $\sim 50 \%$ by calculation from the actin-activated myosin ATPase activities of rats in group A4 (Table V). A similar conclusion can be drawn from analyzing the data within the framework of the "two state" model of cross-bridges $(45,60)$. In this model, force of the isometric twitch and myosin ATPase activity are both defined as functions of the number of cycling crossbridges and of the rate constants of the transition to the forcegenerating state $(f)$ and of the return to the non-force-generating state ( $g 1$ and $g 2$ ). According to this model, force per ATP split is inversely proportional to $g 1$. In our study, the increase in isometric force together with the unchanged myosin ATPase activity in tumor-bearing rats, indicates a decrease in $g 1$ which in the presence of this unchanged ATPase activity suggests an increase in the number of cycling cross-bridges. The constancy of both $V_{\max }$ and total myosin ATPase activity in tumor bearing rats agrees well with the close relationship established between these two parameters in the various cardiac and skeletal muscles $(19,20)$. Since, in A. F. Huxley's model, $V_{\max }$ depends directly upon the $g 2$ rate constant of the exit from the force-generating step, our results suggest that this rate constant was unchanged in tumor-bearing rats.

Despite increased intrinsic myocardial performance, the myosin phenoconversion in tumor-bearing rats was associated with increased economy of force generation (Fig. 2). In fact, the $G$ curvature of the force-velocity relationship is related to the kinetics of myosin cross-bridges $(44,45)$. Fast twitch skeletal muscles and cardiac muscles containing high proportions of $\mathrm{V} 1$ exhibit a low $G$ curvature, whereas the opposite is true for slow twitch skeletal muscles and cardiac muscles with a high proportion of V3 $(37,44,46)$. It has been clearly demonstrated that the latter muscles develop force more economically than the former muscles $(37,44,46,49)$. In our study, the increase in the $G$ curvature is in full agreement with the biochemical demonstration of the presence of functional V3 enzymatic sites. In the context of the A. F. Huxley model, in which the $G$ curvature is a function of the rate constants of cross-bridges cycle, the observed increase in the $G$ curvature could be predicted from the above mentioned alterations in these rate constants.

The radical changes in myocardial myosin phenotype, mechanics and energetics demonstrated here in rats submitted to chronic GH hypersecretion may be due to several factors, including alterations in the working conditions of the heart and/ or direct effects of GH or IGF-I on cardiac myocytes. Although we cannot preclude that a tumor product other than GH (or IGF-I) could be responsible for these changes, this is very unlikely if one consider the effects described by investigators using other GH-secreting cell lines $(15,61)$ or injections of recombinant GH (16). Moreover, we found significant correlations in tumor-bearing rats between plasma $\mathrm{GH}$ and $\mathrm{BW}$, heart weight, percentages of V1 and V3 in the papillary muscles (data not shown). Little is known about a putative direct effect of GH (or IGF-I) on the myocardium. One can simply note that $\mathrm{GH}$ administration in rats is associated with an increase in the myocardial concentration of IGF-I and its mRNA $(62,63)$. Binding sites for IGF-I have also been de- 
tected in neonatal rat myocardium (64). In addition, in humans treatment with recombinant $\mathrm{GH}$ enhances myosin heavy chain mRNA accumulation in skeletal muscle (65).

In conclusion, our mechanical and enzymatic studies consistently showed that during chronic GH hypersecretion, the recruitment of an increased number of functionally active enzymatic sites is responsible for normal overall ATPase activities, normal $V_{\max }$, and increased force development per muscle cross-sectional area. In addition, both the increased $G$ curvature of the force-velocity relationship and the presence of functionally active V3 enzymatic sites strongly suggested that despite the increase in contractile performance, the myocardium functioned at a lower energetic cost. To our knowledge, chronic GH hypersecretion is thus the only case demonstrated to date in which, despite increased force generation, a normal shortening speed and peak power output, changes in the myosin phenotype allow the muscle to function more economically. This situation contrasts with the one created by chronic hemodynamic overload, in which cardiac hypertrophy, myosin phenoconversion and increased economy of force generation are associated with decreased $V_{\max }, A F / s$, and $E_{\max }$. From a thermoenergetic point of view, chronic $\mathrm{GH}$ hypersecretion therefore induces a new pattern of adaptation of the myocardium which might help the heart pump to increase its performance with a better efficiency.

\section{Acknowledgments}

We wish to thank Dr. D. Gourdji for the generous gift of GC cells, Dr. M. T. Bluet-Pajot for her help in hormonal assays, and Drs. L. Rappaport, K. Schwartz, and B. Swynghedauw for their constant interest and support in our work.

This work was supported in part by a grant (Contrat de Recherche Clinique) from the Assistance Publique-Hôpitaux de Paris and by grant HL-33294 from the National Institutes of Health. B. Riou was supported by fellowship grants from the Fonds d'Etude du Corps Médical des Hôpitaux de Paris and the Fondation pour la Recherche Médicale. J. Bertherat was supported by a fellowship grant from the Fondation pour la Recherche Médicale.

\section{References}

1. Nabarro, J. D. N. 1987. Acromegaly. Clin. Endocrinol. 26:481512.

2. Hirsch, E. Z., J. G. Sloman, and F. I. R. Martin. 1969. Cardiac function in acromegaly. Am. J. Med. Sci. 257:1-8.

3. Passa, P., C. Masquet, J. Cophignon, R. Gourgon, and Y. Bouvrain. 1973. Le coeur dans l'acromégalie: étude hémodynamique. Arch. Mal. Coeur. 12:1517-1523.

4. Mather, H. M., M. J. Boyd, and J. S. Jenkins. 1979. Heart size and function in acromegaly. Br. Heart J. 41:697-701.

5. Savage, D. D., W. L. Henry, R. C. Eastman, J. S. Borer, and P. Gorden. 1979. Echocardiographic assessment of cardiac anatomy and function in acromegalic patients. Am. J. Med. 67:823-829.

6. Lie, J. T., and S. J. Grossman. 1980. Pathology of the heart in acromegaly: anatomic findings in 27 autopsied patients. Am. Heart J. 100:41-52.

7. Thuesen, L., S. E. Christensen, J. Weeke, H. Orskov, and P. Henningsen. 1988. A hyperkinetic heart in uncomplicated active acromegaly: explanation of hypertension in acromegalic patients? Acta Med. Scand. 223:337-343.

8. Randall, R. V. 1989. Acromegaly and gigantism. In Endocrinology. L. J. de Groot, editor. W. B. Saunders, Philadelphia. 330-350.

9. Pepine, C. J., and J. Aloia. 1970. Heart muscle disease in acromegaly. Am. J. Med. 48:530-534.
10. Mac Guffin Jr, W. L., B. M. Sherman, J. Roth, P. Gorden, C. R. Kahn, W. C. Roberts, and P. L. Frommer. 1974. Acromegaly and cardiovascular disorders: a prospective study. Ann. Intern. Med. 81:11-18.

11. Jonas, E. A., J. F. Aloia, and F. J. Lane. 1975. Evidence of a subclinical heart muscle dysfunction in acromegaly. Chest. 67:190194.

12. Martins, J. B., R. E. Kerber, B. M. Sherman, M. L. Marcus, and J. C. Ehrhardt. 1977. Cardiac size and function in acromegaly. Circulation. 56:863-869.

13. Smallridge, R. C., S. Rajfer, J. Davia, and M. Schaaf. 1979. Acromegaly and the heart: an echographic study. Am. J. Med. 66:2227.

14. Luboshitzky, R., and D. Barzilai. 1980. Bromocriptine for an acromegalic patient. Improvement in cardiac function and carpal tunnal syndrome. JAMA (J. Am. Med. Assoc.) 244:1825-1827.

15. Penney, D. G., J. C. Dunbar, Jr., and M. S. Baylerian. 1985. Cardiomegaly and hemodynamics in rats with a transplantable growth hormone-secreting tumor. Cardiovasc. Res. 19:270-277.

16. Rubin, S. A., P. Butrick, and A. Malhotra. 1987. Myosin ATPase activity and isoforms in growth hormone induced cardiac hypertrophy. Circulation. 235:937. (Abstr.)

17. Rubin, S. A., T. Nusbickel, T. Sherman, D. Mickle, and S. Melmed. 1986. Cardiac growth and ventricular function after implantation of a growth hormone secreting tumor: a new model of cardiac hypertrophy. J. Am. Coll. Cardiol. 7:54a. (Abstr.)

18. Swynghedauw, B. 1986. Developmental and functional adaptation of contractile proteins in cardiac and skeletal muscles. Physiol. Rev. 66:710-771.

19. Barany, M. 1967. ATPase activity of myosin correlated with speed of muscle shortening. J. Gen. Physiol. 50:197-218.

20. Delcayre, C., and B. Swynghedauw. 1975. A comparative study of heart myosin ATPase and light subunits from different species. Pflügers Arch. 355:39-47.

21. Winegrad, S., G. McClellan, R. Horowits, M. Tucker, L. E. Lin and A. Weisberg. 1983. Regulation of cardiac contractile proteins by phosphorylation. Fed. Proc. 42:39-44.

22. Mahdavi, V., M. Periasamy, and B. Nadal-Ginard. 1982. Molecular characterization of two myosin heavy chain genes expressed in the adult heart. Nature (Lond.). 297:659-664.

23. Pope, B., J. F. Y. Hoh, and A. Weeds. 1980. The ATPase activities of rat cardiac myosin isoenzymes. FEBS (Fed. Eur. Biochem. Soc.) Lett. 118:205-208.

24. Lompré, A. M., J. J. Mercadier, C. Wisnewsky, P. Bouveret, C. Pantaloni, A. d'Albis, and K. Schwartz. 1981. Species- and age-dependent changes in the relative amounts of cardiac myosin isoenzymes in mammals. Dev. Biol. 84:286-290.

25. Litten, R. Z., B. J. Martin, R. B. Low, and N. R. Alpert. 1982. Altered myosin isozyme patterns from pressure-overloaded and thyrotoxic hypertrophied rabbit hearts. Circ. Res. 50:856-864.

26. Hoh J. F. Y., P. A. Mc Grath, and P. T. Hale. 1978. Electrophoretic analysis of multiple forms of rat cardiac myosins: effects of hypophysectomy and thyroxine replacement. J. Mol. Cell. Cardiol. 10:1053-1076.

27. d'Albis, A., C. Pantaloni, and J. J. Bechet. 1979. An electrophoretic study of native myosin isoenzymes and their subunit content. Eur. J. Biochem. 99:261-272.

28. Chizzonite, R. A., and R. Zak. 1984. Regulation of myosin isoenzyme composition in fetal and neonatal rat ventricle by endogenous thyroid hormones. J. Biol. Chem. 259:12628-12632.

29. Lompré, A. M., K. Schwartz, A. d'Albis, G. Lacombe, N. Van Thiem, and B. Swynghedauw. 1979. Myosin isoenzyme redistribution in chronic heart overload. Nature (Lond.). 282:105-107.

30. Mercadier, J. J., A. M. Lompré, C. Wisnewsky, J. L. Samuel, J. Bercovici, B. Swynghedauw, and K. Schwartz. 1981. Myosin isoenzymic changes in several models of rat cardiac hypertrophy. Circ. Res. 49:525-532.

31. Gorza L., P. Pauletto, A. C. Pessina, S. Sartore, and S. Schiaf- 
fino. 1981. Isomyosin distribution in normal and pressure-overloaded rat ventricular myocardium: an immunohistochemical study. Circ. Res. 49:1003-1009.

32. Lompré, A. M., B. Nadal-Ginard, and V. Mahdavi. 1984. Expression of the cardiac ventricular a- and b-myosin heavy chain genes is developmentally and hormonally regulated. J. Biol. Chem. 259:6437-6446.

33. Izumo, S., A. M. Lompré, R. Matsuoka, G. Koren, K. Schwartz, B. Nadal-Ginard, and V. Mahdavi. 1987. Myosin heavy chain messenger RNA and protein isoform transitions during cardiac hypertrophy: interaction between hemodynamic and thyroid hormone-inducad signals. J. Clin. Invest. 79:970-977.

34. Schwartz, K., Y. Lecarpentier, J. L. Martin, A. M. Lompré, J. J. Mercadier, and B. Swynghedauw. 1981. Myosin isoenzymic distribution correlates with speed of myocardial contraction. J. Mol. Cell Cardiol. 13:1071-1075.

35. Ebrecht, G., H. Rupp, and R. Jacob. 1982. Alterations of mechanical parameters in chemically skinned preparations of rat myocardium as a function of isoenzyme pattern of myosin. Basic Res. Cardiol. 77:220-234.

36. Pagani, E. D., and F. J. Julian. 1984. Rabbit papillary muscle myosin isozymes and the velocity of muscle shortening. Circ. Res. 54:586-594.

37. Lecarpentier, Y., L. B. Bugaisky, D. Chemla, J. J. Mercadier, K. Schwartz, R. G. Whalen, and J. L. Martin. 1987. Coordinated changes in contractility, energetics, and isomyosins after aortic stenosis. Am. J. Physiol. 252:H275-H282.

38. Apstein, C. S., Y. Lecarpentier, J. J. Mercadier, J. L. Martin, F. Pontet, C. Wisnewsky, K. Schwartz, and B. Swynghedauw. 1987. Changes in left ventricular papillary muscle performance and myosin composition with aortic insufficiency in rats. Am. J. Physiol. 253:H1005-H1011.

39. Winegrad, S., A. Weisberg, E. R. Lin, and G. McClellan. 1986. Adrenergic regulation of myosin adenosine triphosphatase activity. Circ. Res. 58:83-95.

40. Bluet-Pajot, M. T., D. Durand, F. Mounier, J. F. Léonard, and C. Kordon. 1989. Differential response of lactotrophs and somatotrophs to a joined application of vasoactive intestinal peptide and thyrotrophin-releasing hormone in the rat. J. Neuroendocrinol. 1:135139.

41. Chemla, D., Y. Lecarpentier, J. L. Martin, M. Clergue, A. Antonetti, and P. Y. Hatt. 1986. Relationship between inotropy and relaxation in rat myocardium. Am. J. Physiol. 250:H1008-H1016.

42. Brutsaert, D. L., and V. A. Claes. 1974. Onset of mechanical activation of mammalian heart muscle in calcium and strontium containing solutions. Circ. Res. 35:345-357.

43. Hill, A. V. 1938. The heat of shortening and the dynamic constants of muscle. Proc. R. Soc. Lond. B. Biol. Sci. 126:136-195.

44. Woledge, R. C., N. A. Curtin, and E. Homsher. 1985. Energetic aspects of muscle contraction. Monogr. Physiol. Soc. 41:27-117.

45. Huxley, A. F. 1957. Muscle structure and theories of contraction. Prog. Biophys. Biophys. Chem. 7:255-318.

46. Alpert, N. R., and L. A. Mulieri. 1983. Thermomechanical economy of hypertrophied hearts. In Myocardial Hypertrophy and Failure. N. R. Alpert, editor. Perspect. Cardiovasc. Res. 7:619-630.

47. Winegrad, S., and A. Weisberg. 1987. Isozyme specific modification of myosin ATPase by cAMP in rat heart. Circ. Res. 60:384-392.

48. Mercadier, J. J., Y. Lecarpentier, C. Delcayre, A. M. Lompré, B. Swynghedauw, and K. Schwartz. 1982. Mécanismes biochimiques de l'adaptation myocardique dans l'hypertrophie cardiaque. Arch. Mal. Coeur. 75:1179-1186.

49. Holubarsch, C., R. P. Goulette, R. Z. Litten, B. J. Martin, L. A Mulieri, and N. R. Alpert. 1985. The economy of isometric force development, myosin isoenzyme pattern and myofibrillar ATPase ac- tivity in normal and hypothyroid rat myocardium. Circ. Res. 56:7886.

50. Cueno, R. C., P. Wilmshurst, C. Lowy, G. Mc Gauley, and P. H. Söksen. 1989. Cardiac failure responding to growth hormone. Lancet. i:838-839.

51. Jorgensen, J. O. L., S. A. Pedersen, L. Thuesen, J. Jorgensen, T. Ingemann-Hansen, N. E. Skakkebaek, and J. S. Christiansen. 1989. Beneficial effects of growth hormone treatment in GH-deficient adults. Lancet. $i: 1221-1225$.

52. Thuesen, L., J. S. Christiansen, K. E. Sorensen, J. O. L. Jorgensen, H. Orskow, and P. Henningsen. 1988. Increased myocardial contractility following growth hormone administration in normal man. Dan. Med. Bull. 35:193-196.

53. Thuesen, L., S. E. Christensen, J. Weeke, H. Orskow, and P. Henningsen. 1989. The cardiovascular effects of octreotide treatment in acromegaly: an echocardiographic study. Clin. Endocrinol. 30:619625

54. Gilbert, P. L., R. J. Siegel, S. Melmed, C. T. Sherman, and M. C. Fishbein. 1985. Cardiac morphology in rats with growth hormone-producing tumors. J. Mol. Cell. Cardiol. 17:805-811.

55. Palmiter, R. D., R. L. Brinster, R. E. Hammer, M. E. Trumbauer, M. G. Rosenfeld, N. C. Birnberg, and R. M. Evans. 1982. Dramatic growth of mice that develop from eggs microinjected with metallothionein-growth hormone fusion genes. Nature (Lond.). 300:611-615.

56. Hammer, R. E., R. L. Brinster, M. G. Rosenfeld, R. M. Evans, and K. E. Mayo. 1985. Expression of human growth hormone-releasing factor in transgenic mice results in increased somatic growth. $\mathrm{Na}$ ture (Lond.). 315:413-416.

57. Stier, C. T. Jr., E. A. Cowden, H. G. Frieden, and M. E. M. Allison. 1984. Prolactin and the rat kidney: a clearance and micropuncture study. Endocrinology. 115:362-367.

58. Lei, L. Q., S. A. Rubin, and M. C. Fishbein. 1988. Cardiac architectural changes with hypertrophy induced by excess growth hormone in rats. Lab. Invest. 59:357-362.

59. Hoh, J. F. Y., G. H. Rossmanith, L. J. Kwan, and A. M Hamilton. 1988. Adrenaline increases the rate of cycling of crossbridges in rat cardiac muscle as measured by pseudo-random binary noise-modulated perturbation analysis. Circ. Res. 62:452-461.

60. Brenner, B. 1988. Effect of $\mathrm{Ca}^{2+}$ on cross-bridge turnover kinetics of skinned single rabbit psoas fibers: implications for regulation of muscle contraction. Proc. Natl. Acad. Sci. USA. 85:3265-3269.

61. Prysor-Jones, R. A., and J. S. Jenkins. 1980. Effects of excessive secretion of growth hormone on tissues of the rat, with particular reference to the heart and skeletal muscle. J. Endocrinol. 85:75-82.

62. D'Ercole, A. J., A. D. Stiles, and L. E. Underwood. 1984. Tissue concentrations of somatomedin C: further evidence for multiple sites of synthesis and paracrine or autocrine mechanisms of action. Proc. Natl. Acad. Sci. USA. 81:935-939.

63. Murphy, L. J., G. I. Bell, M. L. Duckworth, and H. G. Friesen. 1987. Identification, characterization, and regulation or rat complementary desoxyribonucleic acid which encodes insulin-like growth factor 1. Endocrinology. 121:684-691.

64. Engelmann, G. L., K. D. Boehm, J. F. Haskell, P. A. Khairallah, and J. Ilan. 1989. Insulin-like growth factors and neonatal cardiomyocytes development: ventricular gene expression and membrane receptor variations in normotensive and hypertensive rats. Mol. Cell. Endocrinol. 63:1-14.

65. Fong, Y., M. Rosenbaum, K. J. Tracey, G. Raman, D. G. Hesse, D. E. Matthews, R. L. Leibel, J. M. Gertner, D. A. Fischman, and S. F. Lowry. 1989. Recombinant growth hormone enhances muscle myosin heavy-chain mRNA accumulation and amino acid accrual in humans. Proc. Natl. Acad. Sci. USA. 86:3371-3374. 